\title{
FACTORS INFLUENCING TEN-YEAR SURVIVAL IN RESECTED STAGES I TO IIIA NON-SMALL CELL LUNG CANCER
}

Nael Martini, MD $^{\mathrm{a}}$

Valerie W. Rusch, MD ${ }^{\mathrm{a}}$

Manjit S. Bains, MD

Mark G. Kris, $\mathrm{MD}^{\mathrm{b}}$

Robert J. Downey, MD

Betty J. Flehinger, $\mathrm{PhD}^{\mathrm{a}}$

Robert J. Ginsberg, MD
Objective: The purpose of this study was to determine (in survivors of 5 years after resection of their lung cancer) whether age, sex, histologic condition, and age have any influence on furthering survival beyond 5 years. Methods: From 1973 to 1989, 686 patients were alive and well 5 years after complete resection of their lung cancers. Survival analysis was carried out with only deaths from lung cancer treated as deaths. Deaths from other causes were treated as withdrawals. Multivariate Cox regression was used to test the relationship of survival to age, sex, histologic condition, and stage. Results: The population in this study had the following characteristics at the time of operation: The male/female ratio was 1.38:1, and the median age was 61 years. The histologic condition of their lung cancer was adenocarcinoma in 412 patients, squamous cell in 244 patients, large cell carcinoma in 29 patients, and small cell carcinoma in 1 patient. The stage of the disease was stage IA in 263 patients, IB in 261 patients, IIA in 12 patients, IIB in 68 patients, and IIIA in 82 patients. The extent of resection was a lobectomy or bilobectomy in 579 patients, pneumonectomy in 55 patients, and wedge resection or segmentectomy in 52 patients. A recurrence or a new lung primary occurrence was considered as failure to remain free of lung cancer. The median follow-up on all patients was 122 months from initial treatment. Of the 686 patients, 26 patients experienced the development of late recurrence and 36 new cancers, beyond 5 years. Overall survival for 5 additional years after a 5-year check point was $\mathbf{9 2 . 4 \%}$. Likewise, survival by nodal status was $\mathbf{9 3 \%}$ for N0 tumors, $\mathbf{9 5 \%}$ for N1 tumors, and $\mathbf{9 0 \%}$ for N2 tumors. Survival by stage was $93 \%$ for stage I tumors and $91 \%$ for stage II or IIIA tumors. Conclusions: In patients with surgically treated lung cancer, neither age, sex, histologic condition, nor stage is a predictor of the risk of late recurrence or new lung cancer. The only prognostic factor appears to be the survival of the patient free of lung cancer for 5 years from the initial treatment, with a resultant favorable outlook to remain well for $\mathbf{1 0}$ or more years. (J Thorac Cardiovasc Surg 1999;117:32-8)
A though there is considerable published information about the natural history of non-small cell lung cancer within the first 5 years after resection, there is relatively little information about the long-term survival of such patients beyond 5 years.

The first retrospective analysis of 10-year survivors after treatment of lung cancer covered the experience at

From the Thoracic Division, ${ }^{a}$ Department of Surgery, and the Thoracic Oncology Section, ${ }^{\mathrm{b}}$ Department of Medicine, Memorial Sloan-Kettering Cancer Center, New York, NY.

Read at the Seventy-eighth Annual Meeting of The American Association for Thoracic Surgery, Boston, Mass, May 3-6, 1998.

Received for publication May 8, 1998; revisions requested June 19,
Memorial Sloan-Kettering Cancer Center from 1949 to 1972. ${ }^{1}$ This previous study investigated the distinguishing characteristics associated with a favorable prognosis after treatment of lung cancer. Not unexpectedly, stage proved to be the most important determinant of long-term survival.

Our current study was prompted by our interest in

1998; revisions received Aug 28, 1998; accepted for publication Sept 2, 1998.

Address for reprints: Nael Martini, MD, 1275 York Ave, New York, NY 10021.

Copyright (C) 1999 by Mosby, Inc.

0022-5223/99 $\$ 8.00+0 \quad \mathbf{1 2 / 6 / 9 4 2 7 9}$ 
Table I. Criteria for diagnosis of multiple lung cancers

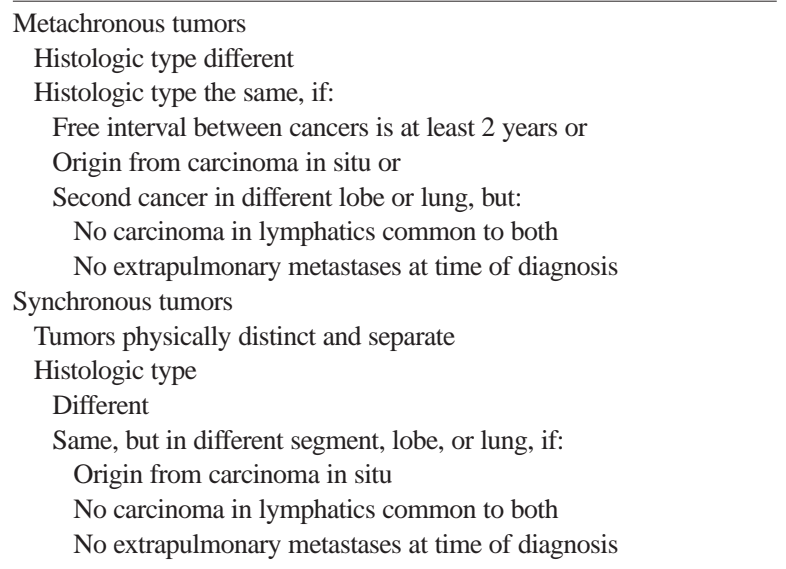

finding out what the risks of recurrence or new lung primary occurrence are in patients who are free of disease 5 or more years from surgical treatment of their initial cancer.

The study focused on the 5-year survivors free of cancer and covered the patients treated by resection for stages I, II, and IIIA disease at our center. Those patients with stage IIIB or IV disease were excluded because they are generally not offered an operation.

The objectives were to determine in survivors of 5 years after resection whether there were differences in survival by lymph node status at initial presentation; whether age, sex, or histologic condition had any influence on furthering survival beyond 5 years; and what the frequency of recurrence, if any, was beyond 5 years.

\section{Patients and methods}

From 1973 to 1989,1490 patients underwent complete resection of lung cancer. Of these, 686 patients (46\%) were seen by us 5 years after the treatment and were free of cancer.

This included patients with pathologic stage I, II, and IIIA tumors and represented $59 \%$ of all patients treated by complete resection at our center during that time period for stage I cancer, $25 \%$ of stage II cancer, and $29 \%$ of stage IIIA cancer.

At thoracotomy, the extent of intrathoracic disease was carefully assessed with particular reference to regional lymph nodes (N1 or N2). A complete resection of all disease in the lung was performed in all patients, and a mediastinal lymph node dissection was performed in most of the patients. Postoperative pathologic staging was done after the resected specimen was reviewed histologically, and all regional nodes were assessed. Histologic type, tumor location, tumor size, histologic clearance at bronchial and vascular resection margins, and visceral pleural involvement were recorded. The surgical and pathologic reports of all patients were reviewed to ensure that the resection was complete.
Table II. Histologic condition at the time of the diagnosis of lung cancer

\begin{tabular}{lc}
\hline \multicolumn{1}{c}{ Cell type } & Number $(\%)$ \\
\hline Adenocarcinoma & $412(60)$ \\
Adenocarcinoma & 328 \\
Bronchoalveolar carcinoma & 84 \\
Squamous carcinoma & $244(36)$ \\
Large cell carcinoma & $29(4)$ \\
Small cell carcinoma & $1(<1)$ \\
Total & $686(100)$ \\
\hline
\end{tabular}

We defined local recurrence as evidence of tumor within the same lung or at the bronchial stump, regional recurrence as clinically or radiologically manifest disease in the mediastinum or in supraclavicular nodes, and distant recurrence as disease in the contralateral lung or outside the hemithorax.

Except in instances where different histologic conditions were present, our guidelines to define second lung primary occurrences were as follows: We recognized a second lung lesion as a new primary even if a tumor of the same histologic condition was found in a different lobe or lung and all regional nodes (N1 or N2) were free of tumor (Table I). ${ }^{3,4}$ In squamous carcinomas, the presence of in situ change in the respective lobar or segmental bronchus suggested a new lung primary occurrence even if found in the same lobe. Because of the frequency of multicentric presentations in bronchoalveolar carcinoma, this subset was excluded from consideration of multiple lung primaries unless the second lesion appeared 2 or more years after the initial resection and no lymph node metastasis was found at the initial surgical treatment or at the time of appearance of the presumed second primary.

To avoid controversy about recurrence or a new lung primary occurrence, we analyzed both groups and considered them failures to remain free of lung cancer.

Survival was calculated by the Kaplan-Meier method, ${ }^{5}$ including postoperative deaths and all lung cancer-related deaths. All other deaths were treated as withdrawals. Differences in survival were determined by the log-rank test and difference in frequency data by the $\chi^{2}$ test. Multivariate Cox regression ${ }^{6}$ was used to test the relationship of survival to age, sex, histologic condition, and stage. Age was treated as a continuous variable; sex was treated as a categoric variable and histologic condition was treated as a categoric variable with 3 classes, which are adenocarcinoma, squamous carcinoma, and others (Table II). Stage was used as an ordinal variable with 3 possible values (Table III).

\section{Results}

There were 398 male patients and 288 female patients. The age range at initial treatment was 19 to 87 years, with a median age of 61 years. There were 120 patients (17\%) who were 70 years of age or older and 17 patients who were younger than 40 years of age. All 
Table III. Pathologic stage after the initial treatment

\begin{tabular}{lcc}
\hline & Number & Total $(\%)$ \\
\hline Stage I & & $524(76)$ \\
IA (T1 N0) & 263 & \\
IB (T2 N0) & 261 & $80(12)$ \\
Stage II & & \\
IIA (T1 N1) & 12 & \\
IIB (T2 N1) & 43 & $82(12)$ \\
IIB (T3 N0) & 25 & \\
Stage IIIA & & \\
IIIA (T3 N1) & 8 & $686(100)$ \\
IIIA (T1-3 N2) & 74 & \\
Total & & \\
\hline
\end{tabular}

patients had a Karnofsky performance status of $80 \%$ or better. $^{7}$

The extent of pulmonary resection consisted of a lobectomy or bilobectomy in $84 \%$ of patients, a pneumonectomy in $8 \%$ of patients, and lesser resections in the remaining patients (Table IV).

The histologic condition was adenocarcinoma in $60 \%$ of patients, squamous cell carcinoma in $36 \%$ of patients, and large cell carcinoma in $4 \%$ of patients (Table II).

On the basis of the new international staging system for lung cancer published in June 1997, ${ }^{7,8} 76 \%$ of the patients had stage I disease; $12 \%$ of the patients had stage II disease, and $12 \%$ of the patients had stage IIIA disease (Table III). Of the 74 patients with stage IIIA (N2) disease, 61 patients were treated initially with complete resection, and 13 patients received induction therapy before complete resection.

Incidence of recurrence or new lung cancers after treatment of the initial cancer. Of 686 patients, 33 patients had recurrences before they survived to 5 years. All 33 patients were rendered disease free. Of these, 20 recurrences were at distant sites, 5 were local recurrences, 7 were regional recurrences, and 1 was unspecified. Fourteen of the distant recurrences were solitary brain metastases, of which 12 were resected and 2 were treated by external radiation therapy only. Two distant recurrences were in the buttock and were treated by resection and postoperative external radiation therapy. Two metastases in the ilium and spine were treated with external radiation therapy. One metastasis in an inguinal node was excised, and 1 metastasis in the lung was treated by intraoperative interstitial implantation of iodine 125 . Two of the local recurrences were in the lung and were treated by resection. One bronchial lung recurrence, 2 chest wall recurrences, and 7 regional metastases into mediastinal or
Table IV. Extent of pulmonary resection at initial

\begin{tabular}{lc} 
treatment & \\
\hline \multicolumn{1}{c}{ Treatment } & Number \\
\hline Lobectomy & 509 \\
Sleeve lobectomy & 12 \\
Bilobectomy & 58 \\
Pneumonectomy & 55 \\
Wedge resection & 45 \\
Segmentectomy & 7 \\
Total & 686 \\
\hline
\end{tabular}

cervical lymph nodes were treated by external radiation therapy. The site of the recurrence or its treatment was not specified in one patient. Of the 33 patients in whom a recurrence had developed and had been rendered disease free before 5 years, 28 patients remained free of cancer, and 5 patients had additional recurrences after 5 years and died despite treatment.

Multiple lung cancers were seen and treated in 28 patients (11 synchronous, 17 metachronous) before they attained their 5-year survival free of disease. All were treated by surgical resection.

Incidence of recurrence and new lung primary after 5 years. There were 36 patients in whom new lung cancers developed after 60 months. Of these, 23 patients have died of the new cancers, 10 patients are alive and well, 2 patients are alive with disease from the new lung cancer, and 1 patient has died of an unrelated cause.

A total of 26 patients had late recurrences after 60 months. Of these, 24 patients have died of the disease, and 2 are alive with the disease. Recurrences were local in 3 patients, regional in 3 patients, local and regional in 2 patients, and distant in 18 patients. Of the distant metastases, 9 metastases were in the brain, 3 metastases were in the lung, 2 metastases were in bone, 2 metastases were in the liver, and 2 metastases were in bones and lungs.

Survival at or after 10 years. Of the 686 patients who had survived 5 years and were free of disease, 369 patients were followed up for an additional 5 years. Of these 289 patients (78\%) were still alive free of cancer, an additional 58 patients (16\%) died subsequently of unrelated causes, 18 patients died of disease, and 4 patients were alive with disease.

Multivariable Cox regression showed that age $(P=$ .70), sex $(P=.37)$, histologic condition $(P=.42)$, stage $(P=.36)$, and the likelihood ratio analysis (significance level, .29) provided evidence that none of the variables used had significantly influenced survival. There was a 




Fig 1. Overall survival (95\% confidence limits at 10 years, $89.9 \%$ to $94.5 \%$ ).

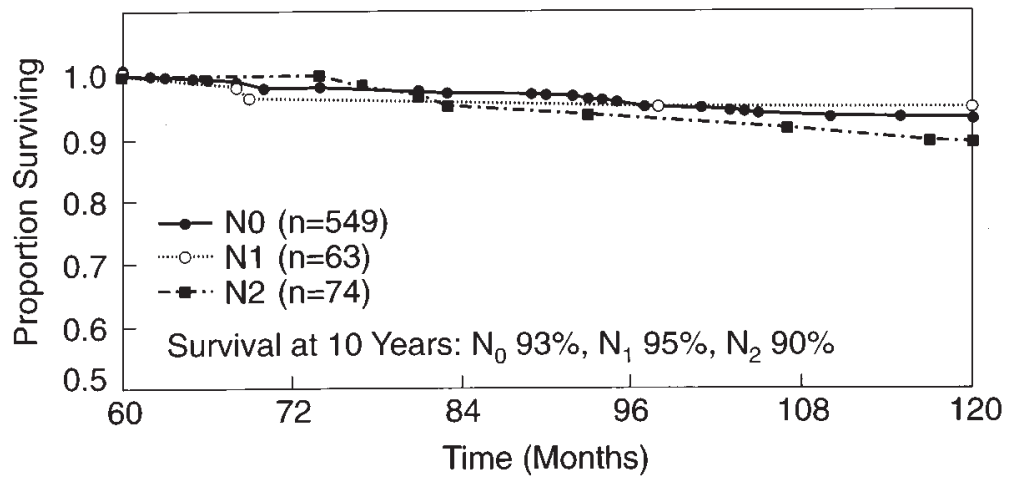

Fig 2. Survival by nodal status.

persistent although somewhat diminishing incidence of new lung primaries despite survivals of up to 20 or more years. However, the incidence of late recurrences diminished more noticeably with time.

The overall 10-year survival (Kaplan-Meier) of the patients who were cancer free at 5 years was $92.4 \%$ (Fig 1). Of the 686 patients, 445 patients $(65 \%)$ were alive and disease free at the time of this reporting, 177 patients $(26 \%)$ had died of unrelated causes, and 60 patients had died of either late recurrence or a new lung primary occurrence plus 4 patients who were alive with disease (total, 9\%). For patients who were alive and well 5 years after treatment of the initial lung cancer, the probabilities of survival beyond 5 years were excellent, irrespective of the nodal status (Fig 2) or stage of disease at initial presentation (Fig 3).

\section{Discussion}

Most reports in the literature on survival after treatment of non-small cell lung cancer are calculated from the date of diagnosis or initial treatment. ${ }^{1,10,12-15}$ Information regarding the risks of recurrence or new lung primary occurrences after a survival free of disease of 5 or more years are sparse and focus mainly on resected stage I lung cancers.

This study focused on patients who were alive with no evidence of disease 5 years after complete resection of lung cancer. It presents estimates of survival and incidence of recurrence and new primary occurrences for patients who are disease free at 5 years. The dependence of survival on age, sex, histologic condition, and initial stage was also analyzed. None of these characteristics had any significant effect on additional survival.

Patients with stage I or II disease are generally offered resection as their primary mode of treatment, and most long-term survivors fall into these 2 groups. Although involvement of the mediastinal nodes (N2) or direct extension of the tumor into the chest wall, diaphragm, or mediastinum (T3) is considered a more advanced 


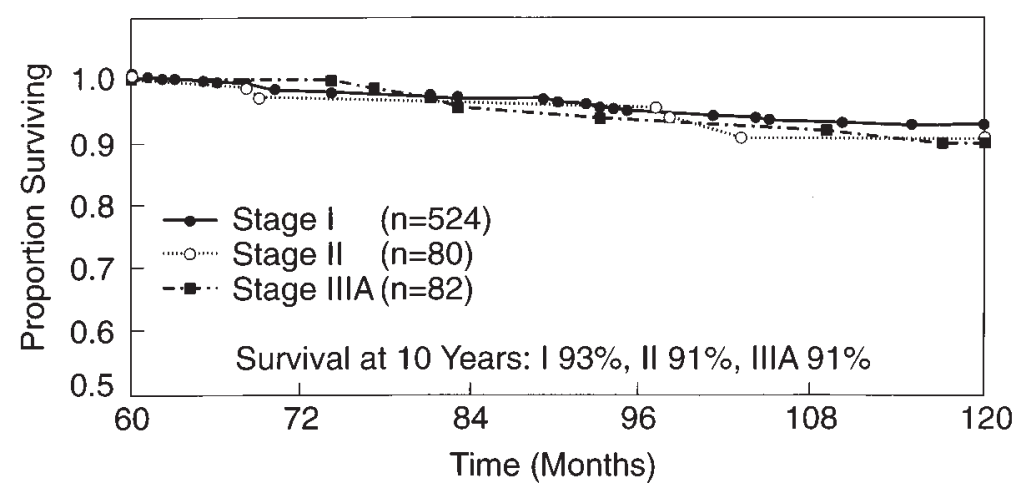

Fig 3. Survival by stage.

stage of disease, 74 patients with stage IIIA (N2) disease and 33 patients with T3 N0-1 M0 tumors were free of cancer at 5 years from their initial surgical treatment.

We have considered a new lung tumor detected after a complete and potentially curative resection of a lung cancer, as a new cancer and not a metastasis, if the free interval between cancers was at least 2 years and all regional nodes (N1 and N2) were negative for tumor. This may have favored in our analysis a greater incidence of new primary occurrences over recurrences. Nevertheless, we felt that any new lung lesion represented failure to control lung cancer.

In the current review, an interesting case was discovered in which recurrence developed 14 years after resection of a stage I bronchoalveolar carcinoma. The patient had multiple pulmonary metastases documented by thoracoscopic biopsy and remains alive 5 years later without treatment.

Although several publications have addressed longterm survival in lung cancer, ${ }^{10-18} 3$ major articles have been recently published on the incidence of late recurrences and new lung primary occurrences. One was the report by the Lung Cancer Study Group, ${ }^{15,19,20}$ the second from the Mayo Clinic, ${ }^{21}$ and the third from our center. ${ }^{2}$ All 3 publications addressed this subject in stage I lung cancers only, whereas the current review addresses, in addition, patients with stage II or IIIa disease, treated by complete resection. Our results indicate that neither age, sex, histologic condition, nor stage is a determinant of risk of late recurrence or new lung cancer. There was no significant difference in the incidence of late recurrence between patients with stage I tumor and those with stage II or III disease. Importantly, recurrences beyond 5 years were usually fatal whereas one third of the patients who experienced the development of new lung primary occurrences survived with treatment.
We recognize that survival from initial diagnosis and treatment of a lung cancer is stage dependent, that surgical treatment is best in stage I or II disease, and that combined modality therapy is essential in the locally advanced tumors. However, despite presumed effective surgical treatment, we are faced with failures to control the cancer mostly because of distant metastases. This is less so in stage I disease and more so in stages II and III tumors. Nevertheless, those patients who remain well for 5 years seem to have a favorable outlook because the vast majority of patients (92\%) do not die of lung cancer thereafter. Because second primary cancers exceed recurrence after 5 years, the need for continued surveillance and consideration of chemoprevention for this group of patients is emphasized.

Our conclusions are that (1) once the patients survive beyond 5 years, there is no difference in survival between N0, N1, and N2 (the disease behaves the same after 5 years); (2) neither age, sex, nor histologic condition further influences the 10-year survival; (3) late recurrences are infrequent; and (4) second primary lung cancers continue to pose a threat although their incidence is also reduced with time.

\section{REFERENCES}

1. Temeck BK, Flehinger BJ, Martini N. A retrospective analysis of 10-year survivors from carcinoma of the lung. Cancer 1984;53: 1405-8.

2. Martini N, Bains MS, Burt ME, et al. Incidence of local recurrence and second primary tumors in resected stage I lung cancer. J Thorac Cardiovasc Surg 1995;109:120-9.

3. Martini N, Ghosh P, Melamed MR. Local recurrence and new primary carcinoma after resection. In: Delarue NC, Eschapasse $\mathrm{H}$, editors. International trends in general thoracic surgery. Vol 1. Philadelphia: WB Saunders; 1985. p. 164-9.

4. Martini N, Melamed MR. Multiple primary cancers. J Thorac Cardiovasc Surg 1975;1:948-56.

5. Kaplan EL, Meier P. Nonparametric estimation from incomplete observations. J Am Stat Assoc 1958;53:457-81. 
6. Cox DR. Regression models and life-tables. J R Stat Soc (B) 1972;34:187-220.

7. Karnofsky DA, Abelman WH, Craver LF, Burchenal JH. The use of the nitrogen mustards in the palliative treatment of carcinoma. Cancer 1948;1:634-56.

8. TNM classification of malignant tumors. Lung. (ICD-O C34). UICC International Union against cancer. New York: Wiley-Liss; 1997. p. 93-7.

9. American Joint Committee on Cancer. AJCC cancer staging manual. Philadelphia/New York: Lippincott-Raven; 1997. p. 127-37.

10. Abbey-Smith R. Evaluation of the long-term results of surgery for bronchial carcinoma. J Thorac Cardiovasc Surg 1981;82:32533.

11. Deslauriers J, Brisson J, Cartier R, et al. Carcinoma of the lung: evaluation of satellite nodules as a factor influencing prognosis after resection. J Thorac Cardiovasc Surg 1989;97:504-12.

12. Kirsh MM, Rotman H, Argenta L, et al. Carcinoma of the lung: results of treatment over the ten years. Ann Thorac Surg 1976; 21:371-7.

13. Volm M, Hahn EW, Mattern J, Muller T, Vogt-Moykopf I, Weber E. Five-year follow-up study of independent clinical and flow cytometric prognostic factors for the survival of patients with non-small cell lung carcinoma. Cancer Res 1988;48:2923-8.

14. Wada H, Fukuse T, Hitomi S. Long-term survival of surgical cases of lung cancer. Lung Cancer 1995;3:269-74.

15. Thomas P, Rubenstein L, Lung Cancer Study Group. Cancer recurrence after resection: T1 N0 non-small cell lung cancer. Ann Thorac Surg 1990;49:242-7.

16. Immerman SC, Vanecko RM, Fry WA, et al. Site of recurrence in patients with stage I and II carcinoma of the lung resected for cure. Ann Thorac Surg 1981;32:23-7.

17. Matthews MJ, Kanhouxve S, Pickrer J, Robinette D. Frequency of residual and metastatic tumor in patients undergoing curative surgical resection for lung cancer. Cancer Chemother Rep 1973; 4:63-7.

18. Shimizu N, Ando A, Date H, Teramoto S. Prognosis of undetected intrapulmonary metastases in resected lung cancer. Cancer 1993:71:3868-72.

19. Gail MH, Eagan RT, Feld R, et al. Prognostic factors in patients with resected stage I non-small cell lung cancer: a report from the Lung Cancer Study Group. Cancer 1984;54:1802-13.

20. The Lung Cancer Study Group. Malignant disease appearing late after operation for T1 N0 non-small-cell lung cancer. J Thorac Cardiovasc Surg 1993;106:1053-8.

21. Pairolero PC, Williams DE, Bergstralh EJ, Piehler JM, Bernatz PE, Payne WS. Postsurgical stage I bronchogenic carcinoma: morbid implications of recurrent disease. Ann Thorac Surg 1984; 38:331-8.

\section{Discussion}

Dr Mark K. Ferguson (Chicago, Ill). This paper is typical of the thoroughly analyzed and data-laden work that has been produced by the Memorial group for the past 25 years. Dr Martini and his colleagues have chosen to address important issues centered around the long-term outcome of 5-year survivors after resection for lung cancer.

As far as I can determine from my review of the literature, these data are unique and quite important. They found that subsequent survival was excellent and was unrelated to stage, nodal status, or sex. The likelihood of cancer recurrence after 5 years was $8 \%$, and after 10 years the recurrence rate was less than $5 \%$. These are important findings that suggest that, from a practical perspective, the 5-year interval is insufficient to declare that a patient with lung cancer may be cured.

I performed a similar analysis of long-term survival of patients receiving operations at the University of Chicago. Our patients were nearly identical to those at Memorial in terms of their age at the time of operation. In our patients, the mean duration of follow-up was similar to that of the Memorial group, 105 months compared with their follow-up of 122 months. Our data support those of the Memorial group in that there was no difference in survival after 5 years, regardless of stage, nodal status, or sex. However, there was a striking difference in the 10-year survival data, which was only $64 \%$ compared with a 10 -year survival in the Memorial data of about $92 \%$.

Their 10-year survival figure is substantially higher than what would be predicted by a life table analysis, which I calculate to be closer to the range of $80 \%$ rather than in excess of $90 \%$. Therefore my first statement concerns the survival calculations you have presented.

They suggest that you have a group of patients who have a history of cancer and who also presumably have an extensive smoking history, but who nevertheless appear to have a better long-term probability of survival than the average population at that age.

My second question addresses the issue of when we can consider a patient with lung cancer to have been cured. There is already considerable controversy about the appropriate interval for patient follow-up and the type of subsequent testing that should be performed for patients with lung cancer. From the data you presented regarding the low incidence of cancer recurrence at 5 years, I submit that this may be a reasonable threshold to permit the declaration of a cancer cure. However, you recommend continued follow-up and further investigation of chemo-prevention techniques for these patients. What specifically do you propose we do about patient follow-up after the 5-year time interval in terms of examination interval and testing, and what information do you have that suggests such follow-up is cost effective?

Dr John R. Benfield (Sacramento, Calif). As you know, one of the ongoing discussions in the treatment of lung cancer is the role of limited resection as compared with standard lobectomy. I do not wish to get into that today except to say that one of the main issues of contention is the significance of local recurrence. In your group of patients who did have recurrence, and I recognize that that is a relatively small number, there must be a subset of patients who had local recurrence only. To what degree did local recurrence only lead to death?

Dr Martini. Dr Ferguson, these patients were not selected by us. They were treated by us and presented to us 5 years later free of cancer. So there was no preselection. For whatever reason, some patients with advanced cancer remain free of disease indefinitely, despite their poor prognosis initially. 
As to the best continued surveillance beyond 5 years, I really do not know the right answer. Cost effectiveness is also an unknown factor. Many believe that it is not cost effective to follow any potentially cured lung cancer after the operation. I do not subscribe to that. I think that once a person has been affected by lung cancer, it is wise to have that patient followed up at least on a yearly interval to rule out recurrence or new cancers.
Dr Benfield, I am not an advocate of a lesser resection than a lobectomy, no matter how small the lesion is and how early it has been detected. The only instance in our review in which there was a failure of control of the disease was in patients who had a second or a third primary lung cancer, when necessarily the surgeon had opted to do a lesser resection. In those patients there was evidence of local recurrence.

\section{ON THE MOVE?}

Send us your new address at least six weeks ahead

Don't miss a single issue of the journal! To ensure prompt service when you change your address, please photocopy and complete the form below.

Please send your change of address notification at least six weeks before your move to ensure continued service. We regret we cannot guarantee replacement of issues missed due to late notification.

\section{JOURNAL TITLE:}

Fill in the title of the journal here.

\section{OLD ADDRESS:}

Affix the address label from a recent issue of the journal here.

\section{NEW ADDRESS: \\ Clearly print your new address here.}

Name

Address

City/State/ZIP

\section{COPY AND MAIL THIS FORM TO:}

Periodical Subscription Services

Mosby, Inc.

11830 Westline Industrial Dr.

St. Louis, MO 63146-3318
OR FAX TO:

314-432-1158

NM Mosby

\section{OR PHONE:}

1-800-453-4351

Outside the U.S., call

314-453-4351 